\title{
As barreiras das mulheres que fazem sexo com mulheres no acesso à saúde no Brasil
}

\author{
The barriers of women who have sex with women in access to health in Brazil \\ Las barreras de las mujeres que tienen sexo con mujeres en el acceso a la salud en Brasil
}

Recebido: 19/11/2021 | Revisado: 28/11/2021 | Aceito: 13/12/2021 | Publicado: 21/12/2021

\author{
Rodrigo Almeida Santiago de Araujo \\ ORCID: https://orcid.org/0000-0001-6484-4294 \\ Universidade Federal de Sergipe, Brasil \\ E-mail: rodrigosantiagomed@gmail.com \\ Raquel de Santana Oliveira \\ ORCID: https://orcid.org/0000-0003-0797-8792 \\ Universidade Tiradentes, Brasil \\ E-mail: rachelsoliveira11@gmail.com \\ Júlia Maria Gonçalves Dias \\ ORCID: https://orcid.org/0000-0001-9145-5534 \\ Universidade Federal de Sergipe, Brasil \\ E-mail: dias_jmg@gmail.com \\ Gabriela de Queiroz Fontes \\ ORCID: https://orcid.org/0000-0003-2014-7686 \\ Universidade Federal de Sergipe, Brasil \\ E-mail: gabrielaqfontes@hotmail.com \\ Thaís Serafim Leite de Barros Silva \\ ORCID: https://orcid.org/0000-0003-1696-6507 \\ Universidade Federal de Sergipe, Brasil \\ E-mail: thaisserafim@hotmail.com \\ Amanda Silvestre Santos Gonçalves \\ ORCID: https://orcid.org/0000-0003-2029-9583 \\ Universidade Federal de Sergipe, Brasil \\ E-mail: amandassgoncalves@gmail.com \\ Alessandra Aleixo Albuquerque \\ ORCID: https://orcid.org/0000-0003-3791-2073 \\ Universidade Federal de Sergipe, Brasil \\ E-mail: alealeixo2011@ hotmail.com
}

\begin{abstract}
Resumo
As mulheres lésbicas e bissexuais são uma minoria invisível quando buscam serviços de atendimento à saúde sexual. Devido a esta invisibilidade dentro de uma cultura milenar de heteronormatividade, a saúde sexual das mulheres lésbicas e bissexuais é, frequentemente, mal conduzida por profissionais da saúde. Partido desse pressuposto, este artigo tem como objetivo identificar as dificuldades na relação médico-paciente e avaliar as barreiras no acesso enfrentadas por mulheres que fazem sexo com mulheres. $\mathrm{O}$ estudo trata-se de uma revisão bibliográfica narrativa, o levantamento das publicações científicas foi nas bases de dados: Lilacs, PubMed, Medline e SciELO. Através das pesquisas, verifica-se que a menor procura pelos serviços de saúde está, muitas vezes, associada à existência de discriminação nos serviços de saúde e ao despreparo dos profissionais para lidar com as especificidades existentes. Além disso, observa-se uma menor frequência de realização de exames ginecológicos, de exames de Papanicolau e de prevenção de câncer de mama entre as mulheres lésbicas e bissexuais. Por fim, faz-se necessário que mais estudos sejam realizados a fim de se colocar em números tais dificuldades e que gerem políticas e estratégias para a melhoria da assistência ginecológica destinada à mulher que faz sexo com mulheres.

Palavras-chave: LGBT; Mulheres que fazem sexo com mulheres; Sexualidade feminina; Infecções sexualmente transmissíveis; Barreiras ao Acesso aos cuidados de saúde.

Abstract

Lesbian and bisexual women are an invisible minority when they're seeking sexual health care services. Due to this invisibility within an ancient culture of heteronormativity, the sexual health of lesbian and bisexual women is often poorly managed by health professionals. Based on this assumption, this article aims to identify the difficulties in the doctor-patient relationship and assess the barriers to access faced by women who have sex with women. The study is a narrative bibliographic review, the survey of scientific publications was in the databases: Lilacs, PubMed, Medline and SciELO. Through research, it appears that the lower demand for health services is often associated with the existence of discrimination in health services and the lack of preparation of professionals to deal with the existing
\end{abstract}


specificities. In addition, there is a lower frequency of gynecological exams, tests and breast cancer prevention among lesbian and bisexual women. Finally, it is necessary that more studies are carried out in order to put such difficulties in numbers and generate policies and strategies to improve gynecological care for women who have sex with women.

Keywords: LGBT; Women who have sex with women; Female sexuality; Sexually transmitted diseases; Barriers to Access to health care.

\section{Resumen}

Las mujeres lesbianas y bisexuales son una minoría invisible cuando buscan servicios de salud sexual. Debido a esta invisibilidad dentro de una antigua cultura de heteronormatividad, la salud sexual de las mujeres lesbianas y bisexuales a menudo es mal gestionada por los profesionales de la salud. Con base en este supuesto, este artículo tiene como objetivo identificar las dificultades en la relación médico-paciente y evaluar las barreras de acceso que enfrentan las mujeres que tienen sexo con mujeres. El estudio es una revisión bibliográfica narrativa, la encuesta de publicaciones científicas se realizó en las siguientes bases de datos: Lilacs, PubMed, Medline y SciELO. A través de la investigación, parece que la menor demanda de servicios de salud se asocia a menudo con la existencia de discriminación en los servicios de salud y la falta de preparación de los profesionales para hacer frente a las especificidades existentes. Además, hay una menor frecuencia de exámenes ginecológicos, pruebas de Papanicolaou y prevención del cáncer de mama entre las mujeres lesbianas y bisexuales. Finalmente, es necesario que se realicen más estudios con el fin de poner tales dificultades en números y generar políticas y estrategias para mejorar la atención ginecológica a las mujeres que tienen sexo con mujeres.

Palabras clave: LGBT; Mujeres que tienen sexo con mujeres; Sexualidad femenina; Enfermedades sexualmente transmisibles; Barreras al acceso a la atención médica.

\section{Introdução}

O direito à saúde no Brasil é fruto da luta do Movimento da Reforma Sanitária, através do SUS, a saúde de maneira ampliada é direito fundamental do ser humano, e é previsto no artigo 196 da Constituição Federal de 1988, o qual determina que:

A saúde é direito de todos e dever do Estado, garantido mediante políticas sociais e econômicas que visem à redução do risco de doença e de outros agravos e ao acesso universal e igualitário às ações e serviços para sua promoção, proteção e recuperação. (Brasil, 1988).

Nesse viés, a Constituição prevê a garantia de atendimento de qualidade a todos, e veda a discriminação por cor, raça, idade, gênero, orientação sexual, opção religiosa ou outros. Da mesma forma, o Código de Ética Médica recomenda que o profissional da Medicina atue em função da saúde do ser humano e da coletividade e exerça seu ofício sem preconceitos de nenhuma natureza. Assim, espera-se que o médico abandone quaisquer julgamentos ao cuidar de seus pacientes, em que pese as diferenças pessoais ou de opinião existentes (Conselho Federal de Medicina, 2010).

Para ser possível o tratamento igualitário, em 2004 o governo federal lançou o programa "Brasil sem Homofobia". O programa busca combater a violência e a discriminação contra lésbicas, gays, bissexuais e transgêneros (LGBT), assim como a promoção da cidadania, sendo um marco histórico na luta pelo direito à dignidade e pelo respeito à diferença, tornando-se o reflexo da consolidação de avanços políticos, sociais e legais tão arduamente conquistados (Brasil, 2004).

Como consequência dos avanços das formulações de políticas públicas, em 2011, foi criada a Política Nacional de Saúde Integral LGBT, formada por diretrizes de governo expressas no Programa Brasil sem Homofobia. A política reconhece a existência de efeitos desumanos dos processos de discriminação, violências e exclusão sobre a saúde da população LGBT e, visando sua superação, constitui-se de um caráter transversal, englobando todas as áreas do Ministério da Saúde relacionadas à produção de conhecimento, participação social, promoção, atenção e cuidado (Brasil, 2013, Silva, 2020).

$\mathrm{O}$ enfrentamento das questões relacionadas à homossexualidade feminina e à sua relação com a saúde, enquanto campo de reivindicações de direitos, emerge no Brasil apenas na virada do século XX para o XXI. As mulheres lésbicas e bissexuais são uma minoria invisível quando buscam serviços de atendimento à saúde sexual. Devido a esta invisibilidade dentro de uma cultura milenar de heteronormatividade, a saúde sexual das mulheres lésbicas e bissexuais é, frequentemente, 
mal conduzida por profissionais da saúde (Carvalho et al, 2013, Clear \& Carryer, 2001, Mcdonald et al 2003, Rondahl et al, 2006, Baker \& Beagan, 2014).

Diante tal cenário, este estudo teve como objetivo identificar as dificuldades na relação médico-paciente e avaliar as barreiras no acesso, utilização dos serviços de saúde e assistência ginecológica direcionada às mulheres que fazem sexo com mulheres.

\section{Metodologia}

O estudo trata-se de uma revisão bibliográfica narrativa. Para Alves-Mazzotti (2002), esse tipo de revisão se refere a criação de uma contextualização para um determinado problema e possibilita a análise das perspectivas presentes na literatura disponível para a construção do referencial teórico da pesquisa.

O levantamento das publicações científicas foi nas seguintes bases de dados: Literatura Latino-americana e do Caribe em Ciências da Saúde (Lilacs), U. S. National Library of Medicine (PubMed), Medical Literature Analysis and Retrieval System Online (Medline) e Scientific Electronic Library Online (SciELO). Inicialmente, para a realização da busca nas principais bases de dados, foram escolhidos os termos: "Minorias Sexuais e de Gênero", "Mulheres que fazem Sexo com Mulheres", "Mulheres Lésbicas" e "Pessoas LGBT". Foram descartados os artigos baseados em opiniões e que não estavam embasados em dados de pesquisa.

\section{Resultados e Discussão}

\section{Condições de Saúde de mulheres lésbicas e bissexuais}

A literatura aponta que a menor procura pelos serviços de saúde está, muitas vezes, associada à existência de discriminação nos serviços de saúde, ao despreparo dos profissionais para lidar com as especificidades existentes, e às dificuldades das mulheres em dialogar sobre a orientação sexual durante o atendimento. (Barbosa, Facchini, 2009). Outro fator preocupante, é a vulnerabilidade às IST's e as práticas preventivas utilizadas por esse grupo populacional. (Albuquerque, et al, 2016).

Para demonstrar em números as dificuldades no acesso à saúde pela população LGBT+, especificamente pelas mulheres lésbicas e bissexuais, o estudo de Pinto et al (2005), conduzido na realidade brasileira, é bastante esclarecedor. Revelou-se que mais da metade das participantes não estava seguindo uma rotina de consultas anuais com um ginecologista, quantitativo também encontrado por Barbosa e Facchini (2009). Chama a atenção o fato de 3,3\% nunca terem visitado um ginecologista e 17,9\% nunca terem realizado o exame de citologia oncótica cervical que detecta alterações epiteliais precocemente e é, portanto, utilizado no rastreio do câncer de colo de útero (Fredrich \& Renner, 2019).

Ademais, há inúmeros empecilhos a serem superados até que seja alcançada a igualdade e a atenção integral em saúde para a comunidade LGBT+. Mesmo assim, as atuais políticas públicas representam não só uma vitória para o SUS, como também admite que a população LGBT+ sofre discriminação e exclusão do processo saúde-doença. (Gomes et al, 2018).

Historicamente, a homossexualidade era considerada uma patologia, inclusive passível de cura, até o ano de 1987, quando a terceira revisão do manual Diagnóstico e Estatístico dos Transtornos Mentais (DSM-3), da Associação Psiquiátrica Americana, retirou a homossexualidade do grupo dos transtornos de personalidade. Em consonância com a evolução dos estudos, em 1993, a OMS anulou a homossexualidade do Código Internacional de Doenças (CID-10).

Na literatura observa-se uma menor frequência de realização de exames ginecológicos, de exames de Papanicolau e de prevenção de câncer de mama entre lés, e que essas mulheres nem sempre procuram cuidados quando necessário ou só o 
realizam em períodos de maiores agravos à sua saúde; e que os profissionais de saúde solicitam menos a realização desses exames a essas mulheres (Barbosa \& Facchini, 2009).

Em uma comparação com as heterossexuais, mulheres lésbicas são dez vezes mais propensas a não fazer e/ ou receber os resultados da citologia e têm quatro vezes mais chances de não se submeter a uma mamografia (Albuquerque et al, 2016; Corrêa-Ribeiro, 2019).

A produção acadêmica motivada pela epidemia do vírus da imunodeficiência humana e da Síndrome da Imunodeficiência Adquirida impulsionou, nas últimas décadas, as pesquisas relativas à sexualidade, configurando novos campos, em especial o dos estudos "gays". No entanto, não é ainda expressiva no Brasil a produção acadêmica sobre a vulnerabilidade das mulheres que se definem como lésbicas às IST's e a AIDS. De forma ainda mais acentuada que a sexualidade feminina heterossexual, o comportamento homossexual feminino tendeu historicamente à invisibilidade no discurso médico-ginecológico. O advento da AIDS contribuiu para a manutenção desta invisibilidade por força da crença de que o "corpo lésbico" seria o único corpo infenso à infecção pela via sexual (Almeida, 2009).

Há uma crença errônea entre a comunidade lésbica de que elas possuem um baixo risco para a transmissão de IST's. Além do HIV, a infecção pelo Papiloma Vírus Humano (HPV) e sua associação com cânceres resultam no aumento de doenças preveníveis e baixos índices de vacinação contra o HPV. Esta crença pode contribuir para níveis maiores de infecção por HPV em mulheres lésbicas e bissexuais, comparados com as heterossexuais. (Waterman \& Voss, 2015, Reiter \& Mcree, 2017, Munson \& Cook, 2016).

Sobre o HPV, o informe técnico do Ministério da Saúde acerca do tema informa que esse é um vírus que apresenta diversos genótipos, dentre os quais 12 são considerados oncogênicos, ou seja, capazes de induzir metaplasias e neoplasias. Os genótipos de maior importância epidemiológica são o 6, 11, 16 e 18. Os dois primeiros são responsáveis por 90\% das verrugas ano-genitais, conhecidas como condilomas acuminados. Já os dois últimos são considerados altamente oncogênicos, sendo o HPV-16 responsável por cerca de 50\% dos cânceres de colo de útero no mundo (Whitworth et al, 2020).

Em vista disso, a vacina contra o papilomavírus humano 6, 11, 16 e 18 foi instituída pelo Ministério da Saúde em 2014, através do Programa Nacional de Imunizações (PNI), como medida de prevenção às neoplasias de colo do útero, vulva, vagina, região anal, pênis e orofaringe. O esquema vacinal eleito para meninas e para meninos é de duas doses (0, 6 meses), sendo a soropositividade atingida próxima de 100\%, garantindo alta eficácia na prevenção contra os HPV referidos. Desde 2017, a vacina é disponibilizada pelo SUS a todas as meninas de 9 a 14 anos, aos meninos de 11 a 14 anos, salvo nos casos de pessoas imunodeprimidas, onde a vacinação se estende para mulheres de 9 até 45 anos e homens de 9 até 26 anos, com esquema de 3 doses (0, 2, 6). (Brasil, 2018).

Outra problemática enfrentada pelas MSM, é a violência. O fenômeno da violência ocorre nas relações homofetivas com a mesma intensidade em que ocorre com casais héteros, no entanto, somada aos aspectos da discriminação e preconceito devido à orientação sexual. (STF, 2013).

A violência contra a população LGBT+ possui diversas formas. Uma delas é a violência sexual. As mulheres lésbicas são as mais atingidas pelo estupro entre a comunidade LGBT+. Em média, 6 lésbicas foram estupradas por dia em 2017, em um total de 2.379 casos registrados, e o que mais chama atenção, é que em $61 \%$ dos casos notificados, a vítima foi estuprada mais de uma vez, segundo levantamento a partir de dados obtidos no Sistema de Informação de Agravos de Notificação (SINAN).

Dessa forma, há então uma diferença da realidade e dos cuidados de saúde entre mulheres heterossexuais e mulheres lésbicas e bissexuais. Ademais, muitas vezes há a quebra de confidencialidade durante consultas e a divulgação da orientação sexual, atitudes inclusive que ferem o Código de Ética Médica. Também, por conta da homofobia institucional, verifica-se a busca de serviços de saúde somente em condições graves, para evitar situações vexatórias e por medo da discriminação. Por 
fim, o cuidado holístico do paciente, que se expande para além das questões sexuais, raramente se concretiza. (Albuquerque et al, 2016; Chinazzo, 2019).

\title{
A relação médico-paciente direcionada às mulheres que fazem sexo com mulheres
}

Em 1995, White e Levinson já consideravam a relação médico-paciente como a base para a boa qualidade no atendimento à mulheres lésbicas e bissexuais:

\begin{abstract}
A maioria das lésbicas desafia estereótipos. É importante perceber que qualquer paciente do sexo feminino pode ser lésbica ou bissexual. Até os médicos saberem a orientação sexual de suas pacientes, é importante usar linguagem livre de suposições heterossexuais. Perguntas como "Que forma de método anticoncepcional você usa?", "Você é casada?" e "Quando foi a última vez que você teve relações sexuais?" estabelecem barreiras para uma paciente lésbica porque eles assumem que ela é heterossexual. Depois de ouvir perguntas como essas, uma lésbica pode reter ou fornecer informações falsas para se proteger de possíveis atitudes negativas de seu médico (White; Levinson, 1995).
\end{abstract}

Embora mulheres lésbicas estejam continuamente adquirindo aceitação dentro da sociedade, muitas continuam expostas a disparidades de saúde significantes e isto aumenta o risco para determinadas condições médicas. Profissionais de saúde também são apontados relatando desconforto com mulheres lésbicas e frequentemente evitando discussões acerca de suas sexualidades, apesar de identificar condições que poderiam afetar a saúde destas mulheres (Austin \& Irwin, 2010, Bonvicini \& Perlin, 2003, Herek et al, 2007).

No contexto médico, pré-determinar que uma paciente é heterossexual é um fator de distanciamento das pacientes lésbicas e bissexuais da assistência à saúde ou de repressão quanto a compartilhar sua sexualidade em consultas médicas. Outrossim, na propedêutica médica temos a orientação sexual dentro do tópico de identificação e aos antecedentes pessoais do paciente por considerar essa informação importante para o entendimento do seu contexto biopsicossocial, bem como para inseri-lo dentro de grupos de cuidado específico de acordo com sua realidade. Dessa forma, ao não adentrar nesse assunto o médico deixa de obter um histórico sexual completo do paciente. (Porto, 2014; Chinazzo, 2019).

Perguntas como "O seu parceiro é homem ou mulher?" e "Como é o seu relacionamento com a pessoa que mora com você?" podem ser realizadas durante a anamnese para diminuir o padrão heteronormativo nas entrevistas. A escuta ativa e a promoção de um ambiente favorável ao diálogo sobre as práticas sexuais devem estar presentes na rotina dos serviços de saúde. Essa abordagem possibilita vínculos e facilita a adesão às tecnologias disponíveis ofertadas pelos profissionais (Brasil, 2019).

Outra lição ensinada para conseguir uma boa anamnese e evitar falhas na comunicação é que o médico deve sempre questionar, em vez de supor circunstâncias e fatos sobre a vida do paciente, especialmente quando de forma precipitada. Tais suposições se baseiam em estereótipos criados em torno da heteronormatividade, e muitas vezes levam o profissional de saúde ao engano e à condução de toda uma investigação e conduta baseadas em uma suposta heterossexualidade. Esse tipo de equívoco exclui a mulher lésbica e principalmente a bissexual, que pode simplesmente omitir suas relações homossexuais, da oportunidade de receber a orientação correta e o acolhimento adequado (Rondahl et al., 2006; Baams et al., 2017).

Nesse contexto, é muito observado na prática médica um grande distanciamento na relação profissional- usuária do sistema de saúde, o que inibe a comunicação sobre as nuances da vida sexual e as demandas relacionadas a isto dentro do consultório (Araújo et al, 2006). Outra grande parcela dessa discussão envolve a homofobia praticada pela equipe, seja por insultos verbais ou negativa do acolhimento, contribuindo de forma substancial para a baixa qualidade do atendimento e o afastamento do público LGBT+ dos serviços de saúde. Em razão disso, o referido grupo atrasa ou evita cuidados necessários quase duas vezes mais (29\%) que os heterossexuais (17\%) (Khalili et al, 2015). 
É nesse cenário que o papel do médico, seja ele especialista em Ginecologia, Sexologia ou um generalista, deve englobar mais do que apenas o cuidado imediato ou focado em patologias. A educação em saúde deve ser incluída com orientações centradas na pessoa com vida sexual ativa e em seus costumes, com o intuito de ajudá-la a reconhecer e minimizar seu risco. Portanto, é importante que o profissional desenvolva a rotina de perguntar a todas as suas pacientes questões sobre sexualidade, ajudando a diminuir o tabu ligado ao diálogo sobre sexo e a desconstruir mitos comuns, como o de que as lésbicas não correm risco de desenvolver câncer de colo de útero e, portanto, não requerem exames regulares de citologia (Pinto et al, 2005; Araújo et al, 2006).

\section{Considerações Finais}

Diante do exposto, foi demonstrado que historicamente, mulheres que fazem sexo com mulheres tiveram seus direitos dirimidos por opiniões e preconceitos cultivados em meio social. No tocante à atenção à saúde não foi diferente.

Nesse contexto, a literatura demonstra ainda uma menor procura por assistência ginecológica por parte de mulheres lésbicas e bissexuais, devido à persistência da discriminação nos serviços de saúde e pela falta de manejo dos profissionais para lidar com as especificidades desse grupo populacional ou por dificuldades das mulheres em abordar sua sexualidade e suas dúvidas na relação médico-paciente.

A literatura ainda é escassa em estudos que contemplem o complexo universo assistencial da saúde ginecológica destas mulheres. Faz-se necessário que mais estudos sejam realizados a fim de se colocar em números tais dificuldades e que gerem políticas e estratégias para a melhoria da assistência ginecológica destinada à mulher que faz sexo com mulheres.

\section{Referências}

Albuquerque, G. A. et al. (2016). Access to health services by lesbian, gay, bisexual, and transgender persons: systematic literature review. BMC International Health and Human Rights, 16(1), 2. 10.1186/s12914-015-0072-9.

Almeida, G. (2009). Argumentos em torno da possibilidade de infecção por DST e Aids entre mulheres que se autodefinem como lésbicas. Physis, Rio de Janeiro, 19(2), 301-331.

Alves-Mazzotti, A. J. A (2002). "Revisão bibliográfica" em teses e dissertações: meus tipos inesquecíveis - o retorno. A bússula do escrever: desafios $e$ estratégias na orientação de teses e dissertações. São Paulo: Cortez, 25-44.

Araújo, M. A. L. et al (2006). Relação usuária-profissional de saúde: experiência de uma mulher homossexual em uma unidade de saúde de referência de Fortaleza, Escola Anna Nery, 10(2), 323-327.

Austin, E. L. \& Irwin J. A. (2010). Health behaviors and health care utilization of southern lesbians. Women's Health Issues, $20,178-184$.

Baker, K. Beagan, B. (2014). Making assumptions, making space: an anthropological critique of cultural competency and its relevance to queer patients. Medical Anthropology Quarterly, 28, 578-598.

Bonvicini, K. A. \& Perlin, M. J. (2003). The same but different: clinician patient communication with gay and lesbian patients. Patient Education and Counseling, 51, 115-122.

Baams, L. Dubas, J. S. \& Van Aken, M. A. G. (2017). Comprehensive Sexuality Education as a Longitudinal Predictor of LGBTQ Name-Calling and Perceived Willingness to Intervene in School, Journal of Youth and Adolescence, 46(5), 931-942.

Barbosa, R. M., \& Facchini, R. (2009). Acesso a cuidados relativos à saúde sexual entre mulheres que fazem sexo com mulheres em São Paulo, Brasil. Cadernos de Saúde Pública, 25(2), 291-300.

Brasil (2013). Ministério da Saúde. Secretaria de Gestão Estratégica e Participativa. Departamento de Apoio à Gestão Participativa. Política Nacional de Saúde Integral de Lésbicas, Gays, Bissexuais, Travestis e Transexuais. Brasília: 1. Ed.

Brasil. Conselho Nacional de Combate à Discriminação. Ministério da Saúde (2004). Brasil Sem Homofobia: Programa de combate à violência e à discriminação contra GLTB e promoção da cidadania homossexual. Ministério da Saúde. Brasília.

Brasil (1988). Constituição da República Federativa do Brasil de 1988. http://www.planalto.gov.br/ccivil_03/constituicao/constituicao.htm

Carvalho, P. M. G. Nobrega, B. S. M. Oliveira, J. L. Almeida, R. O. Abdalla, M. F. T. \& Nichiata, L. Y. I. (2013). Prevention of sexually transmitted diseases by homosexual and bisexual women: a descriptive study. Online Brazilian Journal of Nursing, 12, 931-941.

Clear, G., \& Carryer; J. S. (2001). Dancing in the wings: lesbian women talk about health care. Nursing Praxis in New Zealand, 17, 27-40. 
Research, Society and Development, v. 10, n. 17, e60101723602, 2021

(CC BY 4.0) | ISSN 2525-3409 | DOI: http://dx.doi.org/10.33448/rsd-v10i17.23602

Conselho Federal de Medicina (2010). Código de Ética Médica: Resolução CFM nº1931/09. Brasília: Conselho Federal de Medicina.

Chinazzo, I. R. \& Alegre, P. (2019). Escola de ciências da saúde programa de pós-graduação em psicologia mestrado em psicologia, 6.

Fredrich, É. K. \& Renner, J. D. P. (2019). Cervical cytopathological changes in Pap smear test in the city of Santa Cruz do Sul, Rio Grande do Sul, Brazil. Jornal Brasileiro de Patologia e Medicina Laboratorial, 55, 3.

Gomes, S. M. et al, (2018). O SUS fora do armário: concepções de gestores municipais de saúde sobre a população LGBT. Saúde e Sociedade, 27(4), 11201133 .

Herek, G. M. Chopp, R. \& Strohl, D. (2007). Sexual stigma: putting sexual minority health issues in context. In The Health of Sexual Minorities: Public Health Perspectives on Lesbian, Gay, Bisexual, and Transgender Populations. Meyer I \&amp; Northridge Meds. Springer, New York, 171-208.

Khalili, J. Leung, L. B. \& Diamant, A. L. (2015). Finding the Perfect Doctor: Identifying Lesbian, Gay, Bisexual, and Transgender-Competent Physicians, American Journal of Public Health, 105(6), 1114-1119.

Mato Grosso do Sul. (2020). Violência Doméstica entre Mulheres Lésbicas. http://www.naosecale.ms.gov.br/violencia-domestica-entre-mulheres-lesbicas-2/

Mcdonald, C. Mcintyre, M. \& Anderson, B. (2003). The view from somewhere: locating lesbian experience in women's health. Health Care for Women International, 24, 697-711.

Mcnair, R. (2009). Lesbian and bissexual women's sexual health. Australian Family Physician, 38, 388-393.

Munson, S. \& Cook, C. (2016). Lesbian and bisexual women sexual healthcare experiences. J Clin Nurs, 25(23-24), 3497-3510.

Negreiros, F. R. N. et al, (2019). Saúde de Lésbicas, Gays, Bissexuais, Travestis e Transexuais: da Formação Médica à Atuação Profissional, Revista Brasileira de Educação Médica, 43(1), 23-31.

Pinto, V. M. et al, (2005). Sexually transmitted disease/HIV risk behaviour among women who have sex with women: AIDS, 19(4), 64-69.

Porto, C. C. (2014). Semiologia Médica. (7a ed.), Guanabara Koogan.

Richters, J. et al, (2010). Do women use dental dams? Safer sex practices of lesbians and other women who have sex with women, Sex Health, 7(2), 165-169.

Röndahl, G. Innala, S. \& Carlsson, M. (2006). Heterosexual assumptions in verbal and non-verbal communication in nursing, Journal of Advanced Nursing, 56(4), 373-381.

Silva, A. C. A. Alcântara, A. M. Oliveira, D. C. \& Signorelli, M. C. (2020). Implementação da Política Nacional de Saúde Integral de Lésbicas, Gays, Bissexuais, Travestis e Transexuais (PNSI LGBT) no Paraná. Interface (Botucatu). Brasil. 10.1590/Interface.190568.

Whitworth, H. S. et al, (2020). Efficacy and immunogenicity of a single dose of human papillomavirus vaccine compared to no vaccination or standard three and two-dose vaccination regimens: A systematic review of evidence from clinical trials, Vaccine, 38(6), 1302-1314. 